\title{
Deformation Transfer to Multi-Component Objects
}

\author{
Kun Zhou $^{\dagger} \quad$ Weiwei $\mathrm{Xu}^{\ddagger} \quad$ Yiying Tong§ $\quad$ Mathieu Desbrun ${ }^{\text {II }}$ \\ $\dagger$ Zhejiang University $\quad$ * Microsoft Research Asia $\quad \S$ Michigan State University $\quad{ }^{\mathbb{I}}$ Caltech
}

\begin{abstract}
We present a simple and effective algorithm to transfer deformation between surface meshes with multiple components. The algorithm automatically computes spatial relationships between components of the target object, builds correspondences between source and target, and finally transfers deformation of the source onto the target while preserving cohesion between the target's components. We demonstrate the versatility of our approach on various complex models.
\end{abstract}

Categories and Subject Descriptors (according to ACM CCS): I.3.3 [Computer Graphics]: Geometry-Shape Deformation

\section{Introduction}

Deformation transfer [SP04] has proven to be a valuable technique for remapping rigid or non-rigid animation sequences from one geometry onto another. Despite its ability to transfer entire animation sequences from a surface mesh to another with only little user interaction, its most serious limitation lies in how similar (in shape and topology) the source and target meshes must be. This technique will simply fail if the source and target objects have drastically different topologies, e.g., if the source is a single connected mesh while the target is an object containing multiple components (see Figure 1). However in real-life applications, characters or moving objects usually consist of multiple connected components, as pointed out in a recent paper by Ben-Chen et al. concurrent to our work [BCWG09]. They presented an efficient space deformation transfer technique to handle multi-component objects equipped with polyhedral cages. However, automatic cage generation methods often generate undesirable cages (e.g., fused feet of humanoid models). Moreover, cage-based techniques can also run into numerical issues for thin-shell-like deformation as in Figure 3, where the cage would exhibit significant selfintersection and degeneracy.

In this paper, we remedy these issues by improving upon the surface-based deformation transfer algorithm. Our contribution stems from simple solutions to two main challenges: how to reliably establish correspondences between wildly different source and target meshes without heavy user interaction, and how to maintain the spatial distribution of the multiple components of the target throughout an animation sequence. The versatility of our method allows for a much wider range of applications, as we demonstrate through deformation transfer onto whimsical objects, and retargeting captured motion data onto multi-component targets.

\subsection{Related Work}

Deformation transfer [SP04] provides a simple and efficient way to remap animation sequences from one mesh onto another. This method first builds a correspondence map between the triangles of the source mesh and those of the target mesh by deforming the target into the reference pose of the source through energy minimization. Then an affine transformation matrix (or deformation gradient) is computed from each triangle in the source mesh to its counterpart in the deformed source mesh by appending an additional vertex to each triangle. Finally the transformation matrices are applied to the corresponding triangles in the target mesh, and a linear system (i.e., Poisson equation) is solved to generate the deformed target mesh whose deformation gradients (with respect to the reference target mesh) are as similar as possible to the deformation gradients of the source. The final process is also known as gradient-domain mesh deformation, which has gained a lot of attention in recent years [YZX*04, SCOL*04, BS08].

Since its introduction, the deformation transfer method has been improved and extended by many researchers. Instead of transferring all the gradients of the source deformation, Zayer et al. [ZRKS05] proposed to transfer gradients only at a few key points, and interpolate them to other points of the target with a harmonic function. Botsch et al. [BSPG06] proved that the same transfer results can be achieved without adding an additional vertex to each triangle, speeding up the 


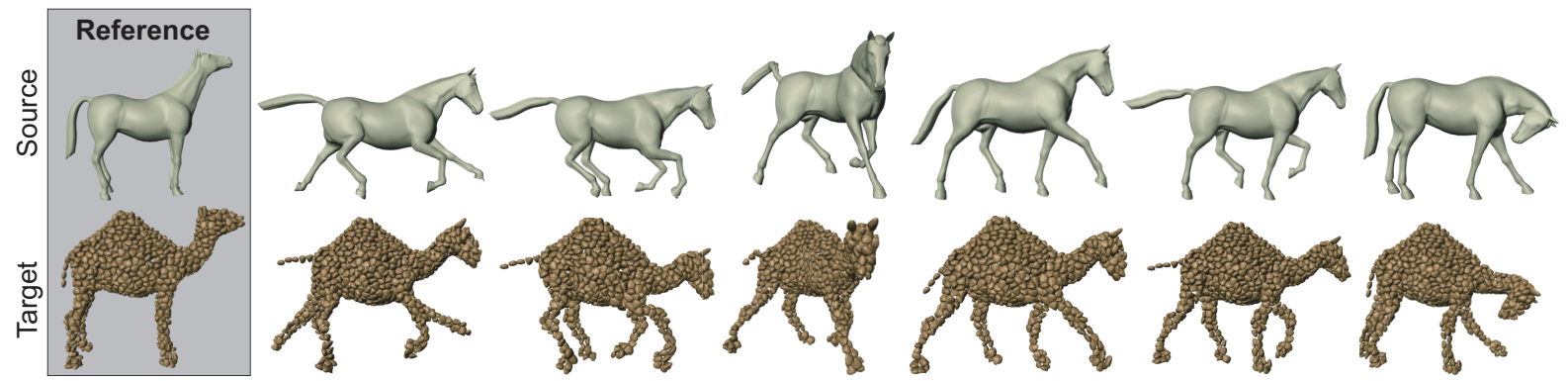

Figure 1: Deformations of a single-component horse are transferred to a pebble camel made of 750 separate stones. Only 47 correspondences are specified. (The pebble camel was inspired by The Thing, a fictional character made of stones in the movie entitled "The Fantastic Four".)

linear system solve. While the original deformation transfer method requires similar reference poses between the source and target meshes, Lee et al. [LWC06] developed a method to remove this restriction. They segment the source and target into the same number of near-rigid components and adjust the orientation of the target components to be similar to the corresponding source components through rigid transformation. More recently, Baran et al. [BVGP09] proposed semantic deformation transfer to preserve the semantic characteristics of the motion instead of its literal deformation.

One serious limitation of Sumner and Popović's method is that it is restricted to single-component meshes. To remove this restriction, Ben-Chen et al. [BCWG09] proposed a spatial deformation transfer technique to handle multiplecomponent meshes and polygon soups. Their technique, however, requires to construct polyhedral cages for the objects. Moreover, some high-frequency details of the source deformation may be lost since the method projects the deformation into a low-dimensional linear subspace. Like Sumner and Popović's original approach, our method is surfacebased, and thus does not suffer from these issues.

\subsection{Algorithm Overview}

Our goal is to extend Sumner and Popović's method to multicomponent meshes. We adopt their approach for the computation of the per-triangle deformation. However, the original method requires a manual specification of a global boundary condition, i.e., an anchor point for each topological component of the mesh to assemble the deformations. Handling objects with multiple parts could thus not be handled for a series of reasons: (1) providing an anchor for each separate component of the target object is difficult; (2) preservation of spatial relationships between components is not addressed; (3) establishing correspondence between the source mesh and the target mesh requires tedious manual work to specify enough marker pairs on each component. To circumvent these issues, we will show that one can employ simple graph theoretical techniques, along with recent developments in mesh deformation that locally preserve shape as best as possible [BS08].
Our algorithm first computes a set of closest vertex pairs to build a graph that encodes the spatial relationship between components. Then both the correspondence algorithm and the deformation transfer algorithm in [SP04] are adapted to take into account the graph connectivity. First, when establishing correspondences between the source and target, we add an energy term to make the components without any user-specified markers deform with the components that have makers. This greatly reduce the manual work to specify markers for each separate component. Note that, unlike in [SP04], where every triangle of the target has a corresponding triangle in the source, not all target triangles may have correspondences in our case. Some components of the target may not have any corresponding triangles in the source. Such components are called orphan components. Also note that, while it is possible to adapt recent automatic algorithms for non-rigid registration [ $\mathrm{WJH}^{*}$ 07,HAWG08,LSP08] to get better correspondences, our current deformation-based approach is simple and easy to implement, and can compute good enough correspondences for the deformation transfer application.

Second, when deforming the target to match the source deformation gradient, we add two new energy terms as soft constraints. The first term tries to preserve the lengths of the graph edges so that the spatial relationship between components is maintained. It also removes the need to provide an anchor for each separate component of the target object. The second term is based on Laplacian coordinates [SCOL*04] and tries to preserve the surface details of the orphan components in the target. Since the two newly added terms are not in quadratic form, the total energy cannot be minimized using a linear solver. Instead, an iterative Gaussian-Newton method is employed - we approximate the two terms as quadratic functions and only need to solve a linear system at each iteration. This optimization scheme works efficiently for all of our experimental data.

One may think that the above two energy terms are unnecessary, and by interpolating the deformation of orphan components from nearby components, the desired deformation can be computed using a linear solver. The assumption of 
this linear approach (i.e., the closest triangle pairs of nearby components have similar deformations), however, is not true in many cases. For example, when the mechanical-like horse shown in Figure 7 is running, two components near to a joint can have different rotations. Enforcing transformation smoothness between the closest triangle pairs may introduce undesirable deformation artifacts (see Figure 7).

\section{Algorithm}

Given a pair of source objects, $\mathbf{S}$ and $\tilde{\mathbf{S}}$, and a target object $\mathbf{T}$ consisting of multiple disconnected components $(\mathbf{T}=$ $\left.\left\{T_{1}, T_{2}, \ldots\right\}\right)$, our goal is to generate a new object $\tilde{\mathbf{T}}$ such that the deformation between $\mathbf{T}$ and $\tilde{\mathbf{T}}$ is analogous to the deformation between $\mathbf{S}$ and $\tilde{\mathbf{S}}$. Note that the source object may also consist of multiple components. For clarity, we will denote by $\mathbf{u}_{i}$ a vertex of $\mathbf{S}$ and by $\tilde{\mathbf{u}}_{i}$ its corresponding vertex in $\tilde{\mathbf{S}}$, while $\mathbf{v}_{i}$ will refer to a vertex of $\mathbf{T}$ and $\tilde{\mathbf{v}}_{i}$ its associated vertex in $\tilde{\mathbf{T}}$ that we will solve for. We will also sometimes indicate with a superscript that a vertex belongs to one of the components of $\mathbf{T} ;$ e.g.. $\mathbf{v}_{i}^{a}$ and $\mathbf{v}_{i}$ will refer to the same vertex, but the superscript stresses the fact that $\mathbf{v}_{i}$ belongs to the component $T_{a}$.

Our approach starts by adding vertices to the source objects. Following [SP04], for each triangle of $\mathbf{S}$ (resp., $\tilde{\mathbf{S}}$ ) with three vertices $\left\{\mathbf{u}_{1}, \mathbf{u}_{2}, \mathbf{u}_{3}\right\}$ (resp., $\left\{\tilde{\mathbf{u}}_{1}, \tilde{\mathbf{u}}_{2}, \tilde{\mathbf{u}}_{3}\right\}$ ), we add a fourth vertex $\mathbf{u}_{4}$ (resp., $\tilde{\mathbf{u}}_{4}$ ) in the direction of the outward normal of the triangle, at a distance from the triangle proportional to the square root of the area. This procedure is designed to make the stretch in the normal direction the geometric average of the principal stretches in the plane. The affine transformation of the source triangle, mapping $\mathbf{u}_{i}$ to $\tilde{\mathbf{u}}_{i}$ for $i=1 \ldots 3$, is computed as:

$$
\mathbf{F}=\tilde{\mathbf{G}} \mathbf{G}^{-1},
$$

where

$$
\begin{aligned}
& \mathbf{G}=\left[\begin{array}{lll}
\mathbf{u}_{2}-\mathbf{u}_{1} & \mathbf{u}_{3}-\mathbf{u}_{1} & \mathbf{u}_{4}-\mathbf{u}_{1}
\end{array}\right] \\
& \tilde{\mathbf{G}}=\left[\begin{array}{lll}
\tilde{\mathbf{u}}_{2}-\tilde{\mathbf{u}}_{1} & \tilde{\mathbf{u}}_{3}-\tilde{\mathbf{u}}_{1} & \tilde{\mathbf{u}}_{4}-\tilde{\mathbf{u}}_{1}
\end{array}\right] .
\end{aligned}
$$

The algorithm then proceeds as follows:

1. Computation of Proximity Pairs between Components: Since $\mathbf{T}$ consists of multiple disconnected components, we start by encoding the spatial relationship between components through a set of proximity pairs. For each pair of components $\left(T_{a}, T_{b}\right)$, we will define as $P_{a, b}$ a selected set of vertex index pairs that represent key spatial adjacencies that subsequent processing will try to preserve during transfer. Note that $P_{a, b}$ may be empty if the associated components are too far away from each other. The computation of $P_{a, b}$ will be described in Section 2.1.

2. Establishment of Correspondences: We assume that the user has provided a small set of markers that indicate point-to-point correspondences between the meshes
$\mathbf{S}$ and T. From these markers, we will deduce correspondences between source triangles and target triangles through a set $M$ :

$$
M=\left\{\left(s_{1}, t_{1}\right),\left(s_{2}, t_{2}\right), \ldots,\left(s_{|M|}, t_{|M|}\right)\right\},
$$

where a pair $\left(s_{m}, t_{m}\right)$ indicates that the target triangle $t_{m}$ should deform like the source triangle $s_{m}$. The computation of $M$ will be explained in Section 2.2. Unlike in [SP04], where every triangle of the target object has a corresponding triangle in the source object, not all target triangles may have correspondences. If the source and target objects have drastically different topologies, enforcing a correspondence to a triangle on the source for each triangle of the target and transferring the deformation will generate unintuitive and unappealing results (see Figure 7). We denote by $H$ the set of "orphan" target triangles (i.e., the ones that do not have correspondences). Note that some components in $\mathbf{T}$ may not contain any corresponding triangles in $M$. We denote by $T_{H}$ the set of these "orphan" components without corresponding triangles.

3. Deformation Transfer: Finally the deformation is transferred through an energy minimization that accounts for the presence of multiple components. Section 2.3 reviews this final process.

We now provide details on the successive steps of our deformation transfer approach.

\subsection{Proximity of Components}

The spatial relationship between the components of the target mesh, encoded as proximity pairs, can be established through the following steps:

- For every pair of components $\left(T_{a}, T_{b}\right)$, find the shortest distance $d_{a, b}$ between them as well as the pair of vertices $\left(v_{i}^{a}, v_{j}^{b}\right)$ corresponding to this shortest distance:

$$
d_{a, b}:=\left\|\mathbf{v}_{i}^{a}-\mathbf{v}_{j}^{b}\right\|=\min _{v_{i} \in T_{a}, v_{j} \in T_{b}}\left\|\mathbf{v}_{i}-\mathbf{v}_{j}\right\| .
$$

- Build a complete graph $G$ with the components as its nodes, where edges are weighted by the shortest distances computed above.

- Compute a minimal spanning tree $K$ (i.e., the minimum weight subgraph) of the graph.

- For each component $T_{a}$, compute its largest distance $d_{a}$ from each of its adjacent components in $K$ :

$$
d_{a}=\max _{\text {edge }(a, b) \in K} d_{a, b}
$$

- For every pair of components $T_{a}$ and $T_{b}$ not connected by an edge in the subgraph $K$, if $d_{a, b} \leq d_{a}+\varepsilon_{a}$ and $d_{a, b} \leq d_{b}+\varepsilon_{b}$, connect $T_{a}$ and $T_{b}$ by adding an edge in $K$ between them. The user-specified thresholds $\varepsilon_{a}$ and $\varepsilon_{b}$ 
define a notion of "close" proximity, controlling how cohesive the target must remain. In our implementation, $\varepsilon_{a}$ (resp., $\varepsilon_{b}$ ) is computed as the maximal edge length of the component $T_{a}$ (resp., $T_{b}$ ) times 1.5 .

The proximity pairs are then easily defined: for two components $T_{a}$ and $T_{b}, P_{a, b}$ is set to nil if these components are not connected by an edge in the subgraph $K$. Otherwise, we set $P_{a, b}$ to be a set initialized with the closest vertex pair $\left(v_{i}^{a}, v_{j}^{b}\right)$; we further add to $P_{a, b}$ any vertex pair $\left(v_{k}^{a}, v_{l}^{b}\right)$ such that $\left\|\mathbf{v}_{k}^{a}-\mathbf{v}_{l}^{b}\right\|<d_{a, b}+\min \left\{\varepsilon_{a}, \varepsilon_{b}\right\}$.

This simple choice of using a few distance-based vertex pairs to encode proximity provides robustness to all sorts of targets while limiting undesirable relative rotations between nearby components, as we will demonstrate in Section 3.

\subsection{Establishing Correspondences}

Finding correspondences between the source and target objects is achieved by deforming the target $\mathbf{T}$ into the static pose of the source $\mathbf{S}$ in a fashion nearly identical to the original deformation transfer approach [SP04]. The deformed vertices of $\mathbf{T}$ that we need to solve for will be called $\mathbf{x}_{i}$, and are only temporarily used in this procedure to establish correspondences.

The user controls the deformation by specifying a set of pairs of source and target vertex indices. Each pair indicates that the target vertex, after deformation, should match the location of the source vertex. Then an iterated closest point algorithm is performed. At each iteration, the target is deformed through an energy minimization, and the set of closest valid vertex-point pairs is updated.

Correspondence Energy We compute the deformed vertices $\mathbf{X}=\left[\begin{array}{l}\mathbf{x}_{1}^{t} \mathbf{x}_{2}^{t} \ldots \\ \ldots\end{array}\right]^{t}$ by minimizing the following energy:

$$
\begin{gathered}
E=w_{S} E_{S}+w_{I} E_{I}+w_{C} E_{C}+E_{R} \\
\text { s.t. } \quad \mathbf{x}_{m_{k}}=\mathbf{m}_{k}, \quad k \in 1 \ldots m
\end{gathered}
$$

where $m_{k}$ is the vertex index on the target mesh for marker $k$, and $\mathbf{m}_{k}$ is the corresponding position of marker $k$ on the source object. The deformation smoothness term $E_{S}$, the deformation identity term $E_{I}$, the closest valid point term $E_{C}$ and the choice of weights are all identical to [SP04]: $E_{S}$ indicates that the transformations of adjacent triangles should be equal, $E_{I}$ is minimized when all transformations are equal to the identity matrix, and $E_{C}$ indicates that the position of each vertex of the target mesh should be equal to the closest valid point on the source mesh. Our only change to the correspondence process lie in the last energy term $E_{R}$. The purpose of this added term is to make the components without any userspecified markers deform along with the components that do have markers, thereby minimizing the need for user-defined correspondences. The $E_{R}$ energy is computed as follows:

- For each component pair $\left(T_{a}, T_{b}\right)$, we find a pair of triangles $\left(t_{i}^{a}, t_{j}^{b}\right)$ for each vertex pair $\left(v_{i}^{a}, v_{j}^{b}\right)$ in $P_{a, b}$, such that

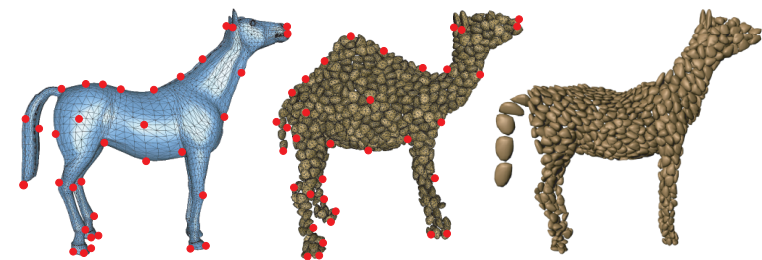

Figure 2: Finding correspondences through deforming the target into the source. From left to right are the source, the target, and the deformed target object. User-specified marker points are shown in red.

$t_{i}^{a} \in \mathcal{N}\left(v_{i}^{a}\right), t_{j}^{b} \in \mathcal{N}\left(v_{j}^{b}\right)(\mathcal{N}($.$) refers to the usual topological$ one-ring), and that the triangles have the largest separation along the normal directions. More precisely, the pair is defined as:

$$
\underset{t_{i}^{a}, t_{j}^{b}}{\arg \max }\left|\mathbf{h} \cdot \mathbf{n}_{t_{i}^{a}}\right|+\left|\mathbf{h} \cdot \mathbf{n}_{t_{j}^{b}}\right|,
$$

where $\mathbf{n}_{t}$ is the normal of triangle $t$, and $\mathbf{h}$ is the vector between the barycenters of the two triangles, $t_{i}^{a}$ and $t_{j}^{b}$.

- For each triangle $t_{i}^{a}$ we found above, we construct a tetrahedron by using one of the three vertices of $t_{j}^{b}$ as the fourth vertex. Similarly, we construct a tetrahedron for triangle $t_{j}^{b}$. However, if triangles $t_{i}^{a}$ and $t_{j}^{b}$ are approximately coplanar in the rest pose of the target object, the two constructed tetrahedra will be degenerate. In this case we simply use $t_{i}^{a}$ 's fourth vertex (that was added in the direction perpendicular to the triangle, see Section 2) as the fourth vertex of both tetrahedra.

- The energy $E_{R}$ is then defined as:

$$
E_{R}=\sum_{a, b} \sum_{\left(t_{i}^{a}, t_{j}^{b}\right)}\left(w_{S}\left\|\hat{\mathbf{F}}_{t_{i}^{a}}-\hat{\mathbf{F}}_{t_{j}^{b}}\right\|^{2}+w_{I}\left\|\hat{\mathbf{F}}_{t_{i}^{a}}-\mathbf{I}\right\|^{2}+w_{I}\left\|\hat{\mathbf{F}}_{t_{j}^{b}}-\mathbf{I}\right\|^{2}\right),
$$

where $\hat{\mathbf{F}}_{t_{i}^{a}}$ indicates the transformation of the newly constructed tetrahedron based on $t_{i}^{a}$.

Note that our goal is to deform the target into the source to establish correspondences. After deformation, the shape of every component as well as the edge length between components may have changed greatly. We thus cannot base our energy on edge length constraint or on the Laplacian constraint, both of which are described in Section 2.3 (as $E_{3}$ and $E_{4}$ ). Our solution, implemented through $E_{R}$, of adding tetrahedra between two components allows for affine transformations of components, and is therefore more effective.

Solving for Correspondences The correspondence energy minimization is achieved via the same two-phase method described in [SP04], consisting in successive linear solves. At each iteration, after the target object is deformed we also need to update the set of the closest valid vertex-point pairs. For each vertex of the deformed target object at the current iteration, we find its closest point on the source object. If 

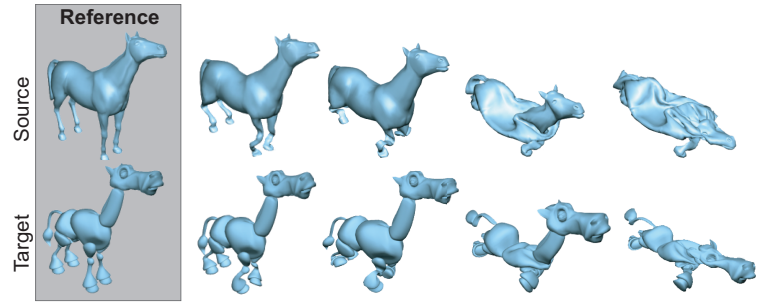

Figure 3: A mechanical-like horse collapsing.

the distance between vertex and point is less than a userspecified threshold and the difference between the vertex's normal and the point's normal is less than 90 degree, we add the vertex and point to the valid set. The whole process converges fast and reaches a minimum after a few iterations (see statistics in Section 3). We then compute the triangle correspondences between the source and the deformed target triangles. For each deformed target triangle, if one or more of its three vertices are among the markers or in the set of the closest vertex-point pairs, it will not be assigned any corresponding source triangle. Otherwise, we label its corresponding source triangle as the one with the smallest distance between the barycenters of the two triangles.

\subsection{Deformation Transfer}

We finally solve for deformed vertex positions $\tilde{\mathbf{V}}=$ $\left[\tilde{\mathbf{v}}_{1}^{t} \tilde{\mathbf{v}}_{2}^{t} \ldots\right]^{t}$ by minimizing the following energy:

$$
E=w_{1} E_{1}+w_{2} E_{2}+w_{3} E_{3}+w_{4} E_{4} .
$$

Each term (explained next) serves a different purpose, while the weights offer control on the overall deformation transfer behavior. We use weights $w_{1}=w_{2}=w_{4}=1.0$ and $w_{3}=0.1$, for all the examples in this paper.

The $E_{1}$ term measures the difference between the source and target transformations (defined in Eq. (1)) :

$$
E_{1}=\sum_{i=1}^{|M|}\left\|\mathbf{F}_{s_{i}}-\mathbf{F}_{t_{i}}\right\|^{2} .
$$

For each triangles in $H$ (i.e., with no corresponding triangle in the source object), $E_{2}$ acts as a regularization, forcing the transformation of this orphan triangle to be close to the transformations of its adjacent triangles (where adjacency is, again, defined as its topological one-ring):

$$
E_{2}=\sum_{t_{i} \in H} \sum_{t_{j} \in \mathcal{N}\left(t_{i}\right)}\left\|\mathbf{F}_{t_{i}}-\mathbf{F}_{t_{j}}\right\|^{2} .
$$

The $E_{3}$ term helps maintain the spatial relationship between components by preserving the edge lengths of the vertex pairs in each $P_{a, b}$ :

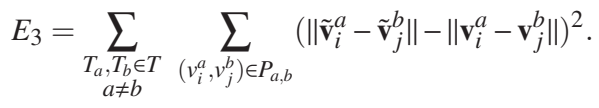

Note that $\left\|\mathbf{v}_{i}^{a}-\mathbf{v}_{j}^{b}\right\|$ is computed on the rest pose of the target object and remains constant. Finally, the components in

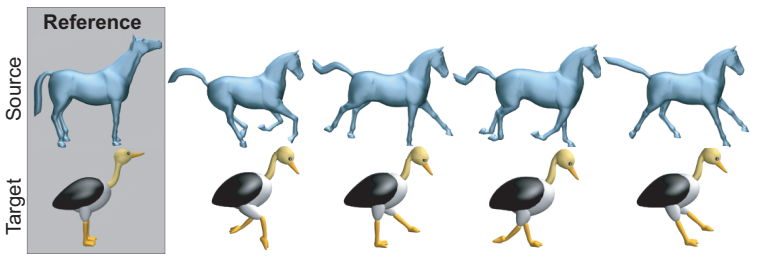

Figure 4: A 9-component bird running like a horse.

\begin{tabular}{|l|c|r|c|}
\hline \multicolumn{1}{|c|}{ Model } & Components & Vertices & Triangles \\
\hline Horse & 1 & 8,431 & 16,843 \\
Woman (source) & 1 & 9,971 & 19,938 \\
Car (source) & 261 & 26,362 & 47,315 \\
Mechanical Horse & 21 & 10,209 & 20,334 \\
Pebble Camel & 750 & 51,000 & 99,000 \\
Bird & 9 & 6,712 & 13,388 \\
Woman (target) & 4 & 12,594 & 25,704 \\
Car (target) & 40 & 28,885 & 57,332 \\
\hline
\end{tabular}

Table 1: Number of components, vertices and triangles for the models used in the paper.

$T_{H}$ do not have corresponding triangles in the source object. Thus, we use Laplacian coordinates [SCOL $\left.{ }^{*} 04\right]$ to preserve the surface details of these components by adding a fourth energy:

$$
E_{4}=\sum_{T_{a} \in T_{H}}\left\|\mathbf{L}_{T_{a}} \tilde{\mathbf{V}}_{T_{a}}-\hat{\delta}\left(\tilde{\mathbf{V}}_{T_{a}}\right)\right\|^{2},
$$

where $\mathbf{L}_{T_{a}}$ is the Laplacian operator matrix computed on the mesh $T_{a}, \tilde{\mathbf{V}}_{T_{a}}$ is a sub-array of $\tilde{\mathbf{V}}$ containing only the vertex positions of component $T_{a}$, and the Laplacian coordinates $\hat{\delta}\left(\tilde{\mathbf{V}}_{T_{a}}\right)$ are defined as in [HSL*06]:

$$
\hat{\delta}\left(\tilde{\mathbf{V}}_{T_{a}}\right)=\frac{\mathbf{L}_{T_{a}} \tilde{\mathbf{V}}_{T_{a}}}{\left\|\mathbf{L}_{T_{a}} \tilde{\mathbf{V}}_{T_{a}}\right\|}\left\|\mathbf{L}_{T_{a}} \mathbf{V}_{T_{a}}\right\|
$$

The total energy $E$ is therefore a nonlinear function of $\tilde{\mathbf{V}}$, but can be efficiently minimized using an iterative GaussNewton method as advocated in [SZT*07]: while $E_{1}$ and $E_{2}$ are quadratic functions of $\tilde{\mathbf{V}}$, repeated quadratic approximations of $E_{3}$ and $E_{4}$ lead to a linear solve for every GaussNewton iteration. Note that the optimization problem requires an additional boundary condition to determine the global translation: this is easily fixed by setting the position for a target vertex, or by specifying another positional constraint such as foot placement.

Note that all energy terms in Eq. (5) were carefully designed, and proved to be necessary in our experiments. In particular, the smoothness term $E_{2}$ is used to propagate the transformations of the triangles with correspondences to those orphan triangles without correspondences. The Laplacian term $E_{4}$ is used to preserve the surface details of orphan components. Omitting $E_{2}$ would leave the transformation matrices of the orphan triangles discontinuous. Thus, the positions of these vertices could not be determined and the orphan triangles would not be deformed accordingly without $E_{2}$. Omitting $E_{4}$ 


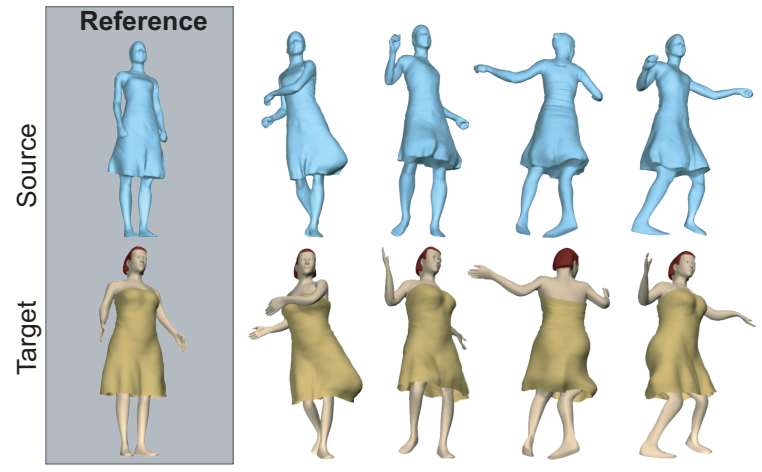

Figure 5: A woman in a dress follows the moves of a dancer.

would leave orphan components under-constrained. The positions of orphan components could thus not be determined.

\section{Results}

We have implemented our deformation transfer algorithm on an Intel Xeon 3.7GHz workstation. We selected a number of examples to demonstrate the versatility of the resulting algorithm. We provide statistics for the models presented in this paper in Table 1 and Table 2, including timings and number of markers needed.

Whimsical Objects The first example shows a deformation of a single-component horse transferred to a robot-looking horse which has 21 components (see Figure 3). Both global and local deformations are nicely reproduced on the new model. Moreover, all components of the model deform consistently and their spatial relationship is well maintained. Note that we constrained a marker vertex on one of the target's feet components to match its correspondence marker on the source. In Figure 4, we transfer the deformations of the horse to a 9-component bird. Although every component intersects with its adjacent components, our algorithm robustly handles the deformation transfer, producing very reasonable results.

Objects with Many Components To test the robustness of our correspondence and transfer algorithm further, we try to transfer the deformations of a horse onto a pebble camel made of 750 separate stones (see Figure 1). In this case, only 47 correspondence markers are specified. Although most components do not have markers, our correspondence algorithm handles them reliably via the extra tetrahedra (added in the energy $E_{R}$ ), and nicely deforms the camel into the horse, establishing satisfactory correspondences. The spatial relationships between components are very well preserved while the deformations are faithfully transferred.

Human body \& Clothes In [VBMP08], complex captured data are encoded as a time-varying single-component mesh. However, transferring deformation from such captured data to other meshes will often involve multiple-component tar-
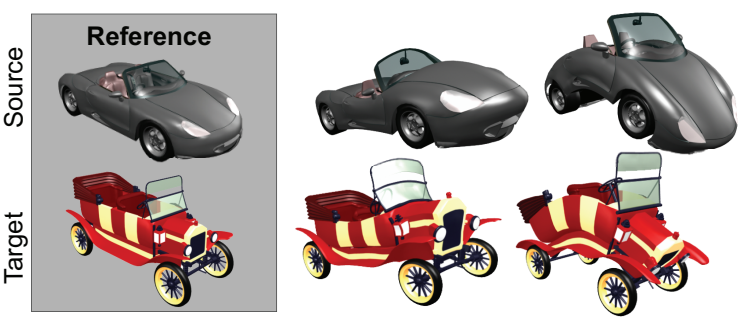

Figure 6: Deformation transfer of multi-component objects.

\begin{tabular}{|l|c|c|r|}
\hline \multicolumn{1}{|c|}{ Example } & \# Markers & Build Corresp. & Transfer \\
\hline Horse/Mec. Horse & 43 & $8.15 \mathrm{~s}$ & $0.424 \mathrm{~s}$ \\
Horse/Bird & 19 & $5.65 \mathrm{~s}$ & $0.439 \mathrm{~s}$ \\
Horse/Pebble Camel & 47 & $54.56 \mathrm{~s}$ & $14.72 \mathrm{~s}$ \\
Woman & 78 & $14.87 \mathrm{~s}$ & $1.83 \mathrm{~s}$ \\
Car & 70 & $32.86 \mathrm{~s}$ & $8.09 \mathrm{~s}$ \\
\hline
\end{tabular}

Table 2: The number of correspondence markers and the timing results (in seconds) for building correspondence and transferring a single frame.

gets: clothes and body meshes (as in Figure 5) are typically given as separate meshes. We show that our approach can transfer animations from multiview silhouettes to multicomponent models created using commercial modeling softwares. Note that in some regions of the target model, the body mesh is very close to the cloth mesh; they both are deformed into the source mesh and find correct correspondences. As a result, these regions of the body are deformed along with the cloth.

Our last example shows that our algorithm can easily handle deformation transfer from one multi-component object to another. As shown in Figure 6, the deformation of a 261component sports car is transferred to a 40-component vintage car model. Note that the algorithm of Section 2 naturally works for multi-component source objects as is.

\section{Conclusion}

We have generalized the deformation transfer technique of [SP04] to handle complex models consisting of arbitrarily many components. We proposed simple methods to establish spatial relationship between components, build correspondences between models with different topologies, and transfer deformation while preserving pairwise component proximity. The resulting algorithm is straightforward to implement.

Although our algorithm works well even in the cloth examples, we cannot guarantee collision-free deformation transfer (see Figure 8). This may be, in certain applications, a serious limitation. For future work, we believe that parts of our algorithm can be easily adopted by as-rigid-aspossible shape interpolation algorithms [ACOL00] to handle multi-component objects. We are also interested in defin- 


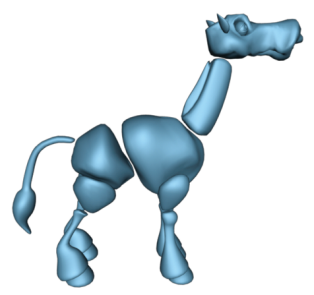

(a) rest pose

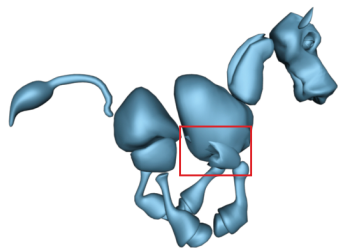

(c) full correspondence method

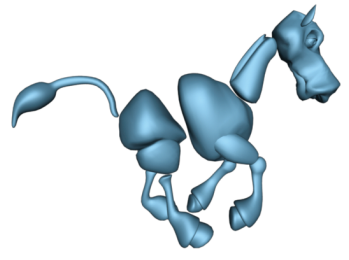

(b) our method

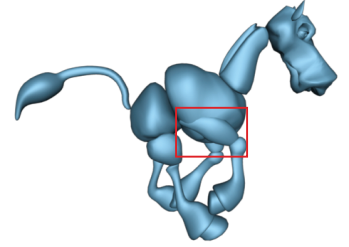

(d) linear method
Figure 7: Comparison on the horse sequence. (a) Rest pose. (b) Transfer result using our method. (c) Transfer result by enforcing a correspondence between a triangle on the source for each triangle of the target. Note the artifacts at the front legs of the horse. (d) Transfer result using a linear method, i.e., without the two terms $E_{3}$ and $E_{4}$ in Eq. (5) and by imposing smoothness constraints on paired tetrahedra found as described in Section 2.2. As closest triangle pairs get different transformations from the source, this method introduces undesirable deformations. The relative distance between components are not well maintained either.

ing and preserving other kinds of spatial relationship between components during deformation transfer; currently we only use edge lengths between vertex pairs, which is inappropriate if the relative distance between components in the source model are supposed to change during deformation.Therefore, extensions could be proven useful.

\section{Acknowledgements}

We would like to thank the anonymous reviewers for their helpful comments. Many thanks to Tianjia Shao and Zhenglong Zhou for their help in video production. This research was partially funded by the NSFC (No. 60825201), the 973 program of China (No. 2009CB320801), the NSF (CCF0811313/0811373, and CMMI-0757106/0757123), and the Open Project Program of the State Key Lab of CAD\&CG, Zhejiang University.

\section{References}

[ACOl00] Alexa M., Cohen-Or D., Levin D.: As-rigidas-possible shape interpolation. In ACM SIGGRAPH (2000), pp. 157-164.

[BCWG09] Ben-Chen M., Weber O., Gotsman C.: Spatial deformation transfer. In Proceedings of SCA'09 (2009).

[BS08] Botsch M., SORKINE O.: On linear variational surface deformation methods. IEEE TVCG 14, 1 (2008), 213-230.
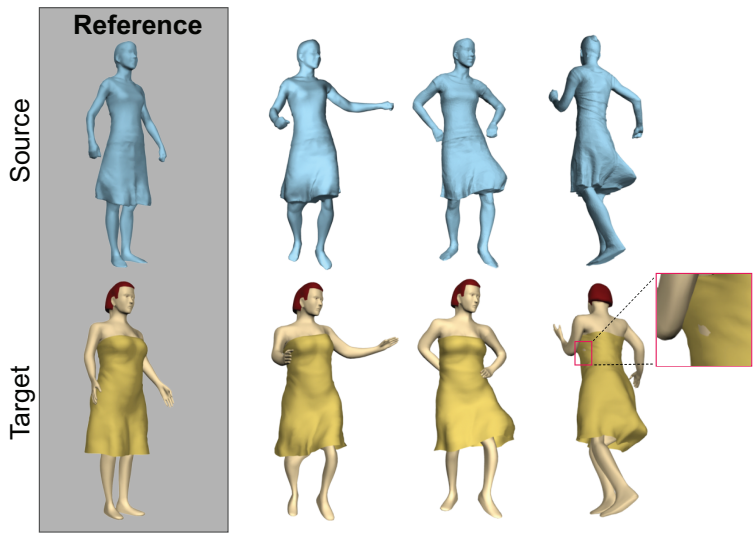

Figure 8: A self-collision example. The samba deformation sequence in [VBMP08] is transferred to the same woman model in Figure 5. We only used 63 markers (compared to the 78 markers used in Figure 5). Self-collisions occur in a few frames of the transfer result.

[BSPG06] Botsch M., Sumner R., Pauly M., Gross M.: Deformation transfer for detail-preserving surface editing. In $\mathrm{Vi}$ sion, Modeling \& Visualization (2006), pp. 357-364.

[BVGP09] Baran I., Vlasic D., Grinspun E., Popović J.: Semantic deformation transfer. ACM TOG 28, 3 (2009), 36.

[HAWG08] HuAng Q.-X., AdAMs B., Wicke M., Guibas L.: Non-rigid registration under isometric deformations. Computer Graphics Forum 27, 5 (2008), 1449-1457.

[HSL*06] Huang J., Shi X., Liu X., Zhou K., Wei L.-Y., TEng S.-H., BaO H., GuO B., Shum H.-Y.: Subspace gradient domain mesh deformation. ACM TOG 25, 3 (2006), 1126-1134.

[LSP08] Li H., Sumner R. W., Pauly M.: Global correspondence optimization for non-rigid registration of depth scans. Computer Graphics Forum 27, 5 (2008), 1421-1430.

[LWC06] LeE T.-Y., WAng Y.-S., Chen T.-G.: Segmenting a deforming mesh into near-rigid components. The Visual Computer 22, 9 (2006), 729-739.

[SCOL*04] SORKINE O., COHEN-OR D., LipMAN Y., ALEXA M., Rössl C., SEIDEL H.-P.: Laplacian surface editing. In Proceedings of SGP'04 (2004), pp. 175-184.

[SP04] SumNeR R. W., Popović J.: Deformation transfer for triangle meshes. ACM TOG 23, 3 (2004), 399-405.

[SZT*07] Shi X., Zhou K., Tong Y., Desbrun M., Bao H., Guo B.: Mesh puppetry: cascading optimization of mesh deformation with inverse kinematics. ACM TOG 26, 3 (2007), 81.

[VBMP08] Vlasic D., Baran I., Matusik W., Popović J.: Articulated mesh animation from multi-view silhouettes. ACM TOG 27, 3 (2008), 97.

[WJH*07] Wand M., Jenke P., HuAng Q.-X., BOKElOH M., Guibas L., SCHILling A.: Reconstruction of deforming geometry from time-varying point clouds. In Proceedings of SGP'07 (2007), pp. 49-58.

[YZX*04] Yu Y., Zhou K., Xu D., Shi X., Bao H., Guo B., SHUM H.-Y.: Mesh editing with poisson-based gradient field manipulation. ACM TOG 23, 3 (2004), 644-651.

[ZRKS05] ZAYER R., RRÖSSl C., KARni Z., SEIDEl H.-P.: Harmonic guidance for surface deformation. Computer Graphics Forum 24, 3 (2005), 601-609. 